MS021.003

Microsymposium

\title{
MOFs - What is in the name?
}

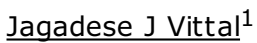

${ }^{1}$ Department Of Chemistry, National University Of Singapore, Singapore, Singapore E-mail: chmjjv@nus.edu.sg

Metal-organic framework (MOF) is a household name in many areas of research including inorganic chemistry, crystal engineering, and materials chemistry. Yaghi introduced this term to describe the gas-storage properties of highly crystalline, thermally stable and porous three-dimensional coordination polymeric structures. The term 'coordination polymers' was originally introduced by Bailar and later popularised by Robson to describe such structures. In the past few years a few attempts have been made to define this terminology. Indeed different terminologies such as porous coordination polymers (PCPs), metal organic materials (MOMs), porous coordination networks (PCNs), hybrid inorganic and organic frameworks, etc have been used in addition to MOFs to describe the non-molecular or extended solid-state structures containing metal ions and organic spacer ligands. In this talk we discuss the origin as well as the pros and cons of using these terminologies in the literature. We will also provide definitions for CPs, COFs and Clathrates.

[1] Li, H. et al. (1999). Nature 402, 276-279.

[2] Biradha, K. et al. (2009). Cryst. Growth. Des. 9, 2969-2970

[3] Batten, S. R. et al. (2012). Pure Appl. Chem. 85, 1715-1724.

Keywords: porous coordination polymers, metal-organic frameworks, terminology 